\title{
Soluble CD14 as a Diagnostic and Prognostic Biomarker in Hematological Patients with Febrile Neutropenia
}

\author{
Sini Korpelainen, ${ }^{1}$ Carina Intke, ${ }^{2}$ Sari Hämäläinen, ${ }^{2}$ Esa Jantunen, ${ }^{2}$ Auni Juutilainen, ${ }^{3}$ and \\ Kari Pulkki ${ }^{1}$ \\ ${ }^{1}$ University of Eastern Finland and Eastern Finland Laboratory Centre, Kuopio, Finland \\ ${ }^{2}$ Kuopio University Hospital, Kuopio, Finland \\ ${ }^{3}$ University of Eastern Finland and Kuopio University Hospital, Kuopio, Finland
}

Correspondence should be addressed to Kari Pulkki; kari.pulkki@uef.fi

Received 10 May 2017; Accepted 28 June 2017; Published 6 August 2017

Academic Editor: Silvia Angeletti

Copyright (c) 2017 Sini Korpelainen et al. This is an open access article distributed under the Creative Commons Attribution License, which permits unrestricted use, distribution, and reproduction in any medium, provided the original work is properly cited.

\begin{abstract}
Objective. Elevated levels of a cell surface glycoprotein, soluble cluster of differentiation 14 (sCD14), have been observed in patients with sepsis. Only scarce data are available on sCD14 in hematological patients with chemotherapy-induced febrile neutropenia. The study aim was to investigate sCD14 as an early biomarker in febrile neutropenia after intensive chemotherapy to detect a rapidly deteriorating clinical course early enough to avoid serious infectious complications. Patients and Methods. This prospective study included 87 adult hematological patients at the start of febrile neutropenia after intensive chemotherapy for acute myeloid leukemia or after autologous stem cell transplantation. The study endpoints were septic shock, severe sepsis, and positive blood culture findings. sCD14 was analyzed from day 0 to day 2, and its prognostic capacity was compared to that of C-reactive protein and procalcitonin. Results. Plasma level of sCD14 predicted the development of septic shock on day $1(p=0.001)$ and day 2 but not the development of severe sepsis or blood culture positivity in hematological patients with chemotherapy-induced febrile neutropenia. Conclusions. Soluble CD14 did not predict an overall complicated course at the early stages of febrile neutropenia. However, it was helpful in predicting the progression of the clinical course of neutropenic fever to septic shock.
\end{abstract}

\section{Introduction}

Patients receiving intensive chemotherapy for hematological malignancies frequently develop febrile neutropenia. They are at high risk of sepsis and septic complications. The onset of septic infection can be insidious, and the outcome may be fatal. An early diagnosis of sepsis is crucial in the prevention of serious complications, and it still remains a challenge for clinicians because of the lack of appropriate diagnostic methods [1]. By definition, demonstrating bacteremia requires blood cultures, but they are time-consuming and often give false-negative results or get microbial contamination [2]. C-reactive protein (CRP) is widely used as an indicator of infection, but it reacts too slowly for prognostic use at the early stages of sepsis [3]. Procalcitonin (PCT) has been studied in neutropenic patients and found to be useful in distinguishing sepsis from noninfectious causes of fever $[4,5]$.
One of the new biomarkers for sepsis is soluble CD14 [6], but there are limited data on its predictive value in febrile neutropenia of adult patients with hematological malignancies.

Cluster of differentiation 14 (CD14) is a cell surface glycoprotein expressed mainly in innate immune response cells such as monocytes, macrophages, neutrophils, and B cells. CD14 recognizes ligands at the cell surface of both gram-negative and gram-positive bacteria and binds to them. It serves as a high-affinity receptor for lipopolysaccharide (LPS) and LPS-binding protein (LBS) complexes. The CD14-LPS-LBS-complex activates the Toll-like receptor 4specific proinflammatory signalling cascade against infectious agents. The complex including CD14 is released from the cell membrane into the circulation creating soluble CD14 (sCD14) [7-9]. Soluble CD14 can also be directly released by hepatocytes [10]. Circulating plasma proteases 
modify soluble CD14 to yet another molecule, soluble CD14 subtype (sCD14-st) or presepsin [7].

Soluble CD14-st (presepsin) binds to bacterial products while circulating in the peripheral blood. It introduces these products to epithelial and endothelial cells, contributing to the activation of the cells against bacteria. Elevated levels of sCD14 have been found in patients with sepsis in several clinical conditions. In previous studies, levels of sCD14 have been associated with sepsis mortality [11-19]. In particular, Burgmann et al. reported that increased levels of serum sCD14 were associated with a high mortality in grampositive sepsis [12].

Also, soluble CD14-st (presepsin) has been considered as a promising biomarker because of its rapid increase at the early stages of sepsis and the availability for bed-side analysis. However, also other than bacteria-related conditions influence the level of sCD14-st, which poses a challenge for its diagnostic use. When used as the only diagnostic method, sCD14-st did not show any advantage over PCT in distinguishing sepsis from nonsepsis $[6,8,20,21]$.

There are only few data on sCD14 in patients with chemotherapy-induced febrile neutropenia at risk for complicated course of sepsis. Urbonas et al. [22] evaluated the predictive value of soluble CD14-st and reported that no association was observed between sCD14-st and bacteremia or sepsis in chemotherapy-induced febrile neutropenia in pediatric patients. According to Urbonas et al., reduced amount of innate immune cells due to chemotherapy may have influenced the results of the study [22]. Also, Olad et al. observed that among neutropenic pediatric cancer patients, sCD14 was not useful in the detection of bacteremia, although sCD14 levels were elevated in blood culturepositive patients, when a clinically detectable source of infection was absent [23].

Data concerning sCD14-st and adult hematological patients at risk for complicated course for febrile neutropenia are especially scarce and controversial. In a study including adult hematological patients with febrile neutropenia, sCD14-st levels were higher in patients with septic shock than in those without it. The study consisted of two cohorts. In the first cohort, the results did not quite gain statistical significance in spite of the positive tendency. Based on the results of the latter cohort, $\mathrm{sCD} 14$-st was an earlier and more sensitive indicator of bacterial infection than PCT, but the number of cases was very small [24].

The aim of this study was to evaluate $\mathrm{sCD} 14$ as a predictor of the progression of febrile neutropenia to sepsis and its complications and to compare $\mathrm{SCD} 14$ with other sepsis biomarkers such as CRP and PCT in hematological patients.

\section{Patients and Methods}

The study population consisted of adult patients treated on the hematology ward of Kuopio University Hospital between November 2009 and November 2012. All patients who were treated for acute myeloid leukemia (AML) or who were autologous stem cell transplant (ASCT) recipients receiving intensive chemotherapy were invited to participate in the study. The final inclusion criteria were fulfilled if a patient
TABLE 1: Characteristics of the study population $(n=87)$.

\begin{tabular}{|c|c|}
\hline \multicolumn{2}{|l|}{ General } \\
\hline Male $(n, \%)$ & $55(63 \%)$ \\
\hline Age (years, median, range) & $61(18-70)$ \\
\hline Age over 60 years $(n, \%)$ & $47(54 \%)$ \\
\hline \multicolumn{2}{|l|}{ Diagnosis $(n, \%)$} \\
\hline AML & $23(26 \%)$ \\
\hline ASCT recipients & $64(74 \%)$ \\
\hline NHL & 43 \\
\hline MM & 18 \\
\hline HL & 3 \\
\hline \multicolumn{2}{|l|}{ Chemotherapy regimen $(n)$} \\
\hline BEAM & 42 \\
\hline HD-MEL & 18 \\
\hline IdAraC-Ida & 9 \\
\hline IAT & 7 \\
\hline MEA & 4 \\
\hline Carmustine-thiotepa & 3 \\
\hline Mito-HDAraC & 2 \\
\hline HDAraC-Ida & 1 \\
\hline BEAC & 1 \\
\hline \multicolumn{2}{|l|}{ Febrile neutropenia } \\
\hline Duration of neutropenia (day, median, range) & $7(4-35)$ \\
\hline Duration of fever (day, median, range) & $3(1-39)$ \\
\hline Positive blood culture finding $(n, \%)$ & $18(21 \%)$ \\
\hline $\begin{array}{l}\text { Gram-negative bacteremia ( } n, \% \text { out of positive } \\
\text { blood culture findings) }\end{array}$ & $3(17 \%)$ \\
\hline $\begin{array}{l}\text { Gram-positive bacteremia ( } n, \% \text { out of positive } \\
\text { blood culture findings) }\end{array}$ & $14(78 \%)$ \\
\hline $\begin{array}{l}\text { Fungal finding ( } n, \% \text { out of positive blood culture } \\
\text { findings) }\end{array}$ & $1(5 \%)$ \\
\hline Complicated course of febrile neutropenia ${ }^{1}(n, \%)$ & $20(23 \%)$ \\
\hline 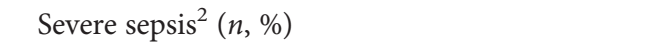 & $8(9 \%)$ \\
\hline Septic shock ${ }^{3}(n, \%)$ & $3(3 \%)$ \\
\hline Fatal outcome $(n, \%)$ & $3(3 \%)$ \\
\hline
\end{tabular}

AML: acute myeloid leukemia; ASCT: autologous stem cell transplant; MM: multiple myeloma; NHL: non-Hodgkin lymphoma; HL: Hodgkin lymphoma; BEAC: carmustine, etoposide, cytarabine, and cyclophosphamide; BEAM: carmustine, etoposide, cytarabine, and melphalan; HD-MEL: high-dose melphalan; Mito-HDAraC: mitoxantrone and high-dose cytarabine; HDAraC-Ida: high-dose cytarabine and idarubicin; IAT: idarubicin, cytarabine, and thioguanine; MEA: mitoxantrone, etoposide, and cytarabine; IdAraC-Ida: intermediate-dose cytarabine and idarubicin. ${ }^{1}$ Febrile neutropenia with a positive blood culture finding and/or development of severe sepsis or septic shock during the period from the onset of febrile neutropenia until the recovery of neutropenia. ${ }^{2}$ Severe sepsis was defined as subset of sepsis with sepsis-induced organ dysfunction. ${ }^{3}$ Septic shock was defined as subset of sepsis with hypoperfusion (systolic arterial pressure $<90 \mathrm{mmHg}$, a mean arterial pressure $<60 \mathrm{mmHg}$, or a reduction in systolic blood pressure of $>40 \mathrm{mmHg}$ from baseline) despite an adequate volume resuscitation in the absence of other causes of hypotension.

had febrile neutropenia. The study population consisted of 87 patients, 23 with AML, and 64 of them were ASCT recipients. The median age was 61 years (range 18-70 years). Further patient characteristics are shown in Table 1. 
All study patients were carefully followed up until recovery of neutropenia (median 7 days, range 4-35 days). Blood pressure, oxygen saturation, respiratory frequency, heart rate, skin temperature, urine output, and fluid intake were followed up. Each patient was daily examined thoroughly for clinical signs and sources of infections. Attention was paid to features indicating the development of severe sepsis or septic shock. Broad spectrum antibiotics were started for all patients after the samples for blood cultures were taken at the onset of fever. Antibacterial treatment was adjusted according to blood culture results, radiological and clinical findings. For patients receiving ASCT for lymphoma, ciprofloxacin-prophylaxis was used. Altogether, 56 out of 87 (64\%) patients received granulocyte colony-stimulating factor in order to shorten the length of neutropenia.

2.1. Study Definitions. A single positive blood culture was considered significant if the microbe was a clinically relevant cause of infection. Common skin contaminants (e.g., coagulase-negative staphylococci) were considered significant only if they were found in two consecutive blood cultures or if there was a concurrent skin or catheter infection.

Febrile neutropenia was defined using the criteria of the Infectious Diseases Society of America [25]. Neutropenia was defined as a neutrophil count less than $0.5 \times 10^{9} / \mathrm{L}$ or a count less than $1 \times 10^{9} / \mathrm{L}$ with a predicted decrease to less than $0.5 \times 10^{9} / \mathrm{L}$. Fever was defined as a single oral temperature of $38.3^{\circ} \mathrm{C}$ or over or a temperature of $38.0^{\circ} \mathrm{C}$ or over for $1 \mathrm{~h}$ or more.

Sepsis and septic shock were defined according to the guidelines of the American College of Chest Physicians Consensus [26, 27]. Sepsis was defined as a syndrome in which systemic inflammatory response was present with infection diagnosed clinically or microbiologically. Septic shock was defined as a subset of sepsis, if hypoperfusion (systolic arterial pressure $<90 \mathrm{mmHg}$, a mean arterial pressure $<60 \mathrm{mmHg}$, or a reduction in systolic blood pressure of $>40 \mathrm{mmHg}$ from baseline) was present despite adequate volume resuscitation in the absence of other causes of hypotension. Severe sepsis was defined as a subset of sepsis with sepsis-induced organ dysfunction [28]. Complicated course of febrile neutropenia was defined as a positive blood culture finding and/or development of severe sepsis or septic shock during the period from the onset of febrile neutropenia until the recovery of neutropenia. In February 2016, new definitions for sepsis and septic shock were announced including the Sequential Organ Failure Assessment (SOFA) and Quick SOFA [29], but in this prospective study design, we used the definitions that were available at the time of the study entry. However, for comparison, also Quick SOFA was retrospectively calculated according to the new sepsis definition [29].

2.2. Blood Cultures. Blood cultures were processed using the automated blood culture system Bactec 9240 (Becton Dickinson, Sparks, MD, USA). The incubation episode was 7 days for both aerobic and anaerobic bottle and 42 days for MYCO F/Lytic bottles.
2.3. Sample Collection and Laboratory Analysis. Blood samples for sCD14, CRP, and PCT analyses were collected at the onset of febrile neutropenia (day 0) and the further samples in the following two mornings (day 1 and day 2). Serum samples were stored frozen at $-70^{\circ} \mathrm{C}$ until analyzed.

The concentration of SCD14 was measured with an ELISA kit (R\&D Systems, Minneapolis, MN, USA). The minimum detectable concentration of SCD14 in this assay was $125 \mathrm{ng} / \mathrm{L}$. The respective intra- and interassay CVs for sCD14 analyses were $10.7 \%$ and $16.0 \%$ for $1285 \mathrm{ng} / \mathrm{L}$ and $1549 \mathrm{ng} / \mathrm{L}$ of sCD14 and $6.1 \%$ and $6.4 \%$ for $1430 \mathrm{ng} / \mathrm{L}$ and $1561 \mathrm{ng} / \mathrm{L}$ of sCD14.

The concentration of CRP was measured with a Konelab $60 \mathrm{i}$ clinical chemistry analyzer (Lab systems CLD, Konelab, Helsinki, Finland) or Cobas 6000 analyzer (Hitachi, Tokyo, Japan). The between-run variations were from $2.3 \%$ to $4.3 \%$. The upper reference limit of serum or plasma CRP of a healthy reference population is $5 \mathrm{mg} / \mathrm{L}$.

Plasma PCT was analyzed with a Cobas 6000 analyzer (Hitachi, Tokyo, Japan). The sensitivity of the assay was $0.06 \mu \mathrm{g} / \mathrm{L}$. The respective within- and between-assay CVs for PCT analyses were $1.4 \%$ and $3.0 \%$ for $0.46 \mu \mathrm{g} / \mathrm{L}$ of PCT and $1.1 \%$ and $2.6 \%$ for $9.4 \mu \mathrm{g} / \mathrm{L}$ of PCT. The reference limit for PCT indicating a possible systemic infection is $0.5 \mu \mathrm{g} / \mathrm{L}$.

2.4. Statistical Analysis. Data analyses were conducted with SPSS version 23 for Windows (SPSS, Chicago, IL, USA). Categorical variables like groups defined by the endpoints were given as absolute counts and percentages. Correlations between variables were analyzed with Pearson's correlation test when appropriate or with nonparametric Spearman's correlation test. The Mann-Whitney $U$ test or KruskalWallis test was used to evaluate the differences in sCD14 concentration levels between patient groups. The difference between sCD14 on day 0 and on day 1 and sCD14 on day 1 and on day 2 was analyzed with related-samples Wilcoxon signed-rank test. Receiver operating characteristic (ROC) curve analysis was performed to compare and describe the diagnostic ability between sCD14, PCT and CRP. A $p$ value of less than 0.05 was considered significant.

2.5. Ethics. The study was conducted in accordance with the current version of the Helsinki Declaration. Informed consent was obtained from all individual participants included in the study. The study has the permission of the Ethical Board of Kuopio University Hospital (100/2006).

\section{Results}

Positive blood cultures were observed in 18 patients with febrile neutropenia (21\%), and three of these patients $(17 \%)$ developed septic shock (two with Enterococcus faecium and one with Pseudomonas aeruginosa). The blood cultures showed gram-negative bacteria in three patients $(17 \%$ out of positive blood culture findings), gram-positive bacteria in 14 patients $(78 \%)$, and a fungus in one patient $(5 \%)$. The gram-negative findings included Escherichia coli $(n=1)$, Klebsiella oxytoca $(n=1)$, and Pseudomonas aeruginosa $(n=1)$. The gram-positive findings included Enterococcus 
faecium $(n=5)$, Staphylococcus epidermidis $(n=3)$, Staphylococcus haemolyticus $(n=2)$, Streptococcus mitis $(n=2)$, Gemella morbillorum $(n=1)$, and Streptococcus salivarius $(n=1)$. The only fungal finding was Candida krusei. Altogether, eight patients (9.2\%) developed severe sepsis, and twenty patients $(23.0 \%)$ fulfilled the criteria for complicated course of febrile neutropenia. Three patients developed septic shock, and two of these three patients died during the hospital stay. Altogether, three patients died during the hospital stay.

When analyzed retrospectively, 7/8 patients with severe sepsis had 1 point in quick SOFA scoring not predicting high risk for in-hospital mortality. Six of these patients were treated at the intensive care unit (ICU), and two of them developed septic shock leading to the death of one of them. Only 1/8 patients received 3 quick SOFA points predicting high risk for in-hospital mortality. This patient developed septic shock and died at the ICU.

The distributions of some of the continuous variables were not normal, so nonparametric tests were used. Data were expressed as medians with interquartile ranges or ranges from minimum to maximum. In all patients, the median serum sCD14 concentration (interquartile range) was $1428(1088-1888 \mathrm{ng} / \mathrm{L}), 1548$ (1084-1992 ng/L), and $1626(1179-2005 \mathrm{ng} / \mathrm{L})$ on days 0,1 , and 2 , respectively. Median CRP level (interquartile range) was $38(19-69 \mathrm{mg} / \mathrm{L})$, 73 (41-119 mg/L), and 103 (45-178 mg/L) on days 0,1 , and 2 , respectively, and the median serum PCT concentration (interquartile range) was $0.138(0.090-0.212 \mu \mathrm{g} / \mathrm{L}), 0.185$ $(0.109-0.408 \mu \mathrm{g} / \mathrm{L})$, and $0.208(0.104-0.547 \mu \mathrm{g} / \mathrm{L})$ on days 0 , 1 , and 2 , respectively. There was no statistically significant increase in the level of sCD14 concentration from day 0 to day 1 or from day 1 to day 2 .

sCD14 concentration did not correlate with CRP or PCT concentration on day 0 , day 1 , or day 2 . The duration of neutropenia did not correlate with sCD14 on any day. Age, sex, comorbidities, or the type of hematological malignancy had no statistically significant association with the levels of sCD14 on days 0,1 , or 2 . sCD14 level had a slight positive correlation with the amount of leukocytes on day 2 (Pearson's correlation coefficient 0.233 with $p$ value 0.036 for leukocytes).

The mean and median of sCD14 concentration on days 1 and 2 in those patients who developed septic shock were higher in comparison to those in patients who did not develop septic shock (Table 2, Figures 1 and 2). The values on day 1 were the highest. The patient with the highest levels of sCD14 (3110 ng/L, $4150 \mathrm{ng} / \mathrm{L}$, and $3730 \mathrm{ng} / \mathrm{L}$ on days 0,1 , and 2, resp.) developed septic shock, dying during the hospital stay on the 12th day after the onset of the fever. The second patient who developed septic shock had levels of sCD14 $1890 \mathrm{ng} / \mathrm{L}, 2250 \mathrm{ng} / \mathrm{L}$, and $2060 \mathrm{ng} / \mathrm{L}$ on days 0,1 , and 2, respectively. He recovered from sepsis. The third patient had a rising value of $\mathrm{sCD} 14$ on days 0 and 1 (1340 ng/L-2550 ng/L). The patient died before day 2; blood samples were not collected on that day.

Although the levels of sCD14 were higher in patients with clinical course progressing to septic shock than in those not with clinical course progressing to septic shock, the patients
TABLE 2: The levels of soluble cluster of differentiation 14 (sCD14), procalcitonin (PCT), and C-reactive protein (CRP) from day 0 to day 2 after the onset of febrile neutropenia in patients with or without septic shock. The data are expressed as medians (minimum-maximum).

\begin{tabular}{cccc}
\hline & $\begin{array}{c}\text { Septic shock } \\
(n=3)\end{array}$ & $\begin{array}{c}\text { Without septic } \\
\text { shock }(n=84)\end{array}$ & $p$ value \\
\hline sCD14 (ng/L) & & & \\
Day 0 & $1888(1337-3108)$ & $1415(349-2734)$ & 0.176 \\
Day 1 & $2550(2249-4146)$ & $1533(436-2746)$ & 0.001 \\
Day 2 & $2895(2058-3731)$ & $1583(655-2776)$ & 0.044 \\
PCT $(\mu \mathrm{g} / \mathrm{L})$ & & & \\
Day 0 & $0.678(0.138-28.6)$ & $0.135(0.037-1.74)$ & 0.049 \\
Day 1 & $1.07(0.891-40.9)$ & $0.180(0.029-28.9)$ & 0.007 \\
Day 2 & $2.91(1.44-4.38)$ & $0.199(0.036-26.9)$ & 0.027 \\
CRP (mg/L) & & & \\
Day 0 & $69(5-212)$ & $37(5-286)$ & 0.640 \\
Day 1 & $226(48-327)$ & $73(9-357)$ & 0.200 \\
Day 2 & $287(231-342)$ & $95(7-367)$ & 0.032 \\
\hline
\end{tabular}

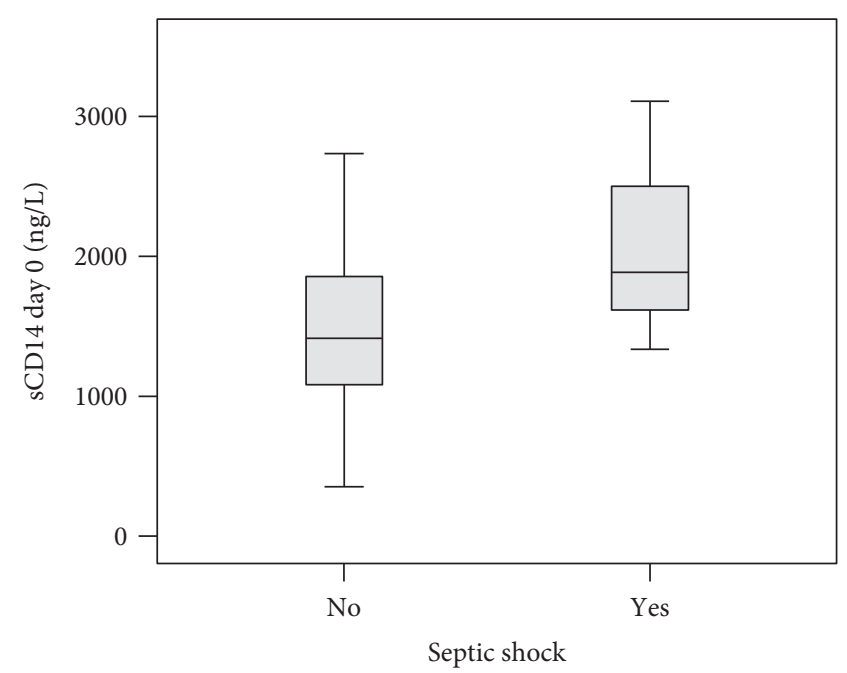

FIgURE 1: Boxplot graph showing sCD14 concentration levels on day 0 in patients with and without developing septic shock ( $p$ value 0.176 ). The horizontal bold line represents median, the box interquartile range, and the end of the lines minimum and maximum values.

with positive and negative blood culture findings had similar levels of sCD14 (Table 3), whether the microbe was grampositive or gram-negative (data not shown). In comparison, plasma levels of CRP on day 1 and PCT on day 0, day 1, and day 2 were higher in blood culture-positive than in blood culture-negative patients.

Furthermore, sCD14 levels at the beginning of febrile neutropenia were not associated with the development of severe sepsis (Table 4). In comparison, elevated levels of CRP on day 1 and day 2 and of PCT from day 0 to day 2 were associated with the development of severe sepsis.

However, sCD14 predicted septic shock on day 1 (with area under the curve (AUC) $0.959,95 \%$ CI $0.902-1.000$, 


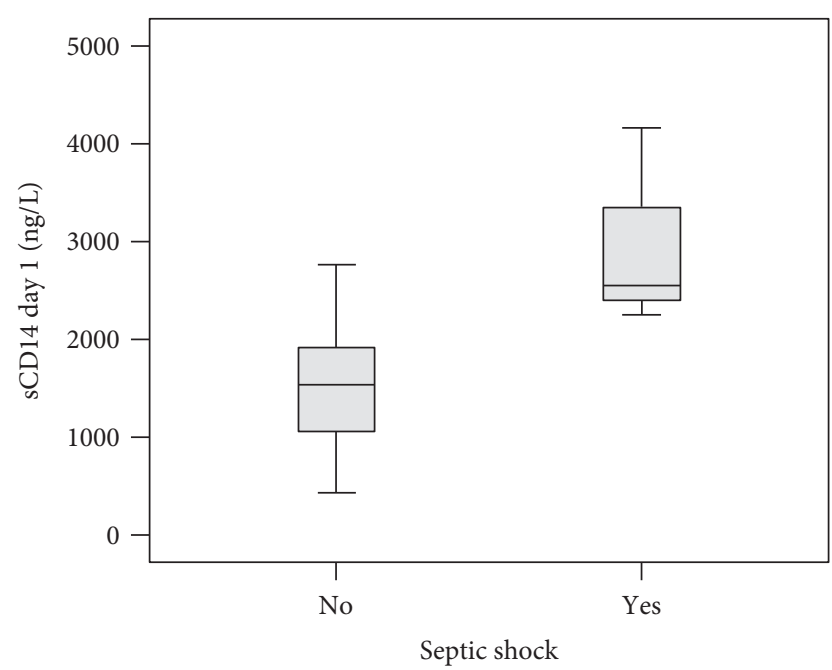

FIGURE 2: Boxplot graph showing sCD14 concentration levels on day 1 in patients with and without developing septic shock ( $p$ value 0.001 ). The horizontal bold line represents median, the box interquartile range, and the end of the lines minimum and maximum values.

TABLE 3: The levels of soluble cluster of differentiation 14 (sCD14), procalcitonin (PCT), and C-reactive protein (CRP) from day 0 to day 2 after the onset of febrile neutropenia in patients with or without blood culture positivity. The data are expressed as medians (minimum-maximum).

\begin{tabular}{|c|c|c|c|}
\hline & $\begin{array}{c}\text { Positive blood } \\
\text { culture finding }(n=18)\end{array}$ & $\begin{array}{c}\text { Negative blood } \\
\text { culture finding }(n=69)\end{array}$ & $p$ value \\
\hline \multicolumn{4}{|c|}{ sCD14 (ng/L) } \\
\hline Day 0 & $1345(349-3108)$ & $1454(506-2734)$ & 0.557 \\
\hline Day 1 & $1251(436-4146)$ & $1578(572-2746)$ & 0.407 \\
\hline Day 2 & $1174(998-3731)$ & $1665(655-2776)$ & 0.059 \\
\hline \multicolumn{4}{|c|}{$\operatorname{PCT}(\mu \mathrm{g} / \mathrm{L})$} \\
\hline Day 0 & $0.182(0.058-28.6)$ & $0.122(0.037-1.74)$ & 0.043 \\
\hline Day 1 & $0.519(0.075-40.9)$ & $0.163(0.029-2.68)$ & 0.001 \\
\hline Day 2 & $0.405(0.073-26.9)$ & $0.172(0.036-6.13)$ & 0.004 \\
\hline \multicolumn{4}{|c|}{$\mathrm{CRP}(\mathrm{mg} / \mathrm{L})$} \\
\hline Day 0 & $47(5-286)$ & $37(5-245)$ & 0.349 \\
\hline Day 1 & $98(14-347)$ & $68(9-357)$ & 0.038 \\
\hline Day 2 & $129(28-367)$ & $88(7-342)$ & 0.097 \\
\hline
\end{tabular}

and $p$ value 0.007 ) showing a higher AUC for sCD14 than that for CRP or PCT in regard to the development of septic shock (Figure 3).

\section{Discussion}

In this three-year prospective study, soluble CD14 did not show any significant association with blood culture positivity or with severe sepsis, but high levels of soluble CD14 on the first and second days of neutropenic fever were associated with the development of septic shock in neutropenic hematological patients who had received intensive chemotherapy.
TABLE 4: The levels of soluble cluster of differentiation 14 (sCD14), procalcitonin (PCT), and C-reactive protein (CRP) from day 0 to day 2 after the onset of febrile neutropenia in patients with or without severe sepsis. The data are expressed as medians (minimum-maximum).

\begin{tabular}{|c|c|c|c|}
\hline & Severe sepsis $(n=8)$ & No severe sepsis $(n=79)$ & $p$ value \\
\hline \multicolumn{4}{|c|}{ sCD14 (ng/L) } \\
\hline Day 0 & $1315(674-3108)$ & $1432(349-2734)$ & 0.580 \\
\hline Day 1 & $1914(869-4146)$ & $1532(436-2746)$ & 0.098 \\
\hline Day 2 & $1639(998-3731)$ & $1605(655-2776)$ & 0.573 \\
\hline \multicolumn{4}{|c|}{ PCT $(\mu \mathrm{g} / \mathrm{L})$} \\
\hline Day 0 & $0.216(0.138-28.6)$ & $0.122(0.037-1.74)$ & 0.044 \\
\hline Day 1 & $0.981(0.154-40.9)$ & $0.176(0.029-28.9)$ & 0.017 \\
\hline Day 2 & $1.44(0.208-26.9)$ & $0.189(0.036-21.1)$ & 0.028 \\
\hline \multicolumn{4}{|c|}{$\mathrm{CRP}(\mathrm{mg} / \mathrm{L})$} \\
\hline Day 0 & $64(5-212)$ & $36(5-286)$ & 0.237 \\
\hline Day 1 & $143(48-327)$ & $68(9-357)$ & 0.037 \\
\hline Day 2 & $231(129-367)$ & $86(7-342)$ & 0.009 \\
\hline
\end{tabular}

The strengths of this study were a homogenous study population of neutropenic hematological adult patients, precise timing of sampling, and the prospective data collection. The main limitation of this study was the small number of patients with septic shock or death. The focus in our study was in pre-emptive patient care, including the aim of early recognition of sepsis. Therefore, the old definition for sepsis, also as the only definition enforced during the study enrolment, was applied $[26,27]$. Retrospectively, we also evaluated Quick SOFA for sepsis definition, but limitations were recognized, as also described by Sprung et al. [30], who critically reviewed the new definition for sepsis based on the problems met in early sepsis recognition and in retrospective derivation for the SOFA score [30]. According to Sprung et al., "the new definitions discard the sepsis spectrum." Our findings indicate that neutropenic sepsis may be one of the sepsis types that are discarded.

Several new biomarkers have been studied for their ability to predict the development of febrile neutropenia to sepsis, but so far, none of them has shown to be superior to traditional diagnostic methods and, therefore, has not been implemented in routine clinical use. Previously, we have discussed the possible impact of the lack of neutrophils on biomarker levels in hematological patients after intensive chemotherapy. The precise influence of neutropenia on the kinetics of a biomarker is usually indeterminate [3, 31-38]. In the present study, a slight but statistically significant positive correlation was observed between the leukocyte count and the levels of sCD14, possibly partly accounting for the overall weak associations between sCD14 levels with the outcomes, as in the presence of low leukocyte count also low levels of sCD14 levels are encountered. Chemotherapeutic agents also influence the function and number of other leukocytes and, therefore, other sources of sCD14 production. This may have further weakened the association of sCD14 levels with blood culture positivity in hematological patients after intensive chemotherapy. In addition to leukocytes, hepatocytes also produce sCD14 $[10,14]$. There are also 


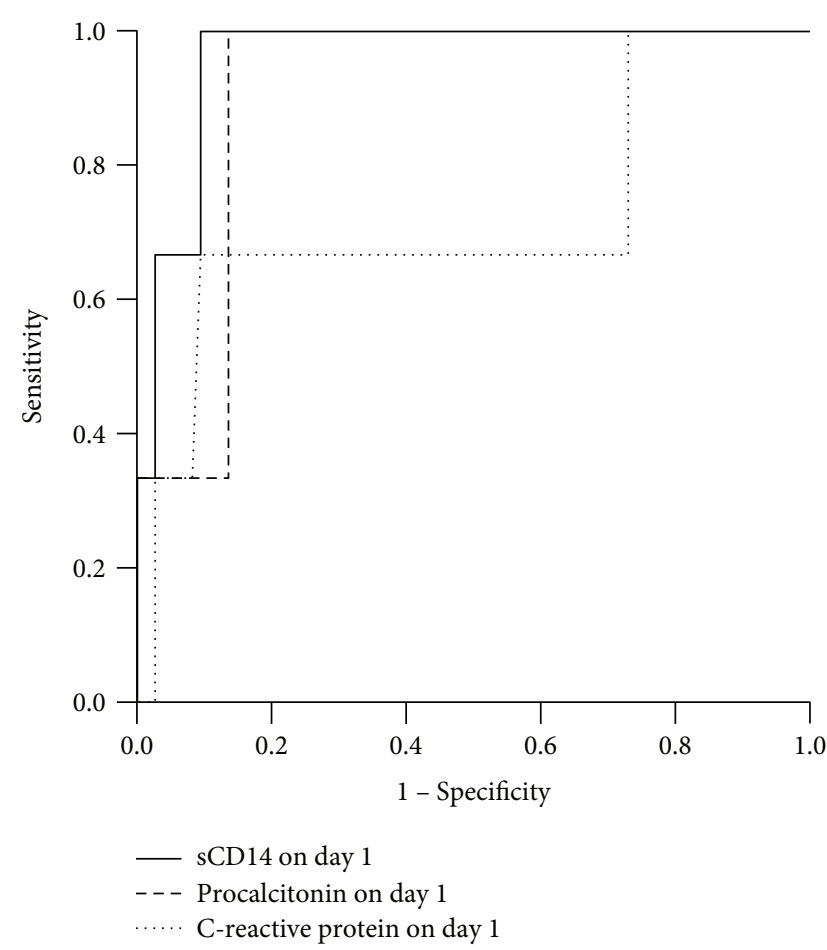

FIGURE 3: Receiver operating characteristic (ROC) curve analysis comparing sCD14 (continuous line), procalcitonin (sparsely dotted line) and C-reactive protein (CRP) (densely dotted line) predicting the development of septic shock on d1. For sCD14, the area under the curve (AUC) was 0.959 (95\% CI 0.902-1.000) with SD 0.029 and $p$ value 0.007 to differentiate patients developing septic shock. For procalcitonin, the AUC was 0.910 (0.819-1.000) with SD 0.047 and $p$ value 0.017 . For CRP, the AUC was 0.716 (0.354-1.000) with SD 0.186 and $p$ value 0.202 (nonsignificant), respectively.

genetic variations in CD14 expression, which influence mCD14 as well as sCD14 levels in serum, but the impact of CD14 expression on the clinical picture of septic infection is unclear [18].

As soluble CD14 is an acute phase protein introducing bacterial products to leukocytes and other cells at the early stage of an infection, it may prevent lethal side effects of bacterial products at the late phase of an infection [17, 39]. High concentrations of soluble CD14 as well as its subtype are also associated with impaired kidney function, which may explain the elevated values in patients with septic shock [40-44]. In combination with other diagnostic methods, sCD14 concentration may be helpful to find neutropenic hematological patients at high risk for potentially lethal septic shock, but further studies in larger patient groups are needed to confirm these preliminary findings.

Sepsis as such is a complicated and multifactorial process with a plethora of potential biomarkers. Typically, sepsis biomarkers are affected by the type of pathogen, comorbidities, genetic variation, medication, and host response. The search for a new biomarker to detect sepsis remains active as there is a constant need to improve the current diagnostic methods in clinical use. The recognition of septic infections is crucial at the onset of neutropenic fever to guide the therapy and to improve the outcome. There is a need to study additional factors, such as the role of underlying diseases, chemotherapeutic regimens, and the influence of neutropenia on blood levels and kinetics of a specific biomarker in patients with chemotherapy-induced neutropenia.

In conclusion, soluble CD14, measured in the beginning of febrile neutropenia after intensive chemotherapy in hematological patients, appeared to be a helpful biomarker in predicting progression of the clinical course to septic shock. However, it did not predict blood culture positivity or severe sepsis and was inferior to PCT and CRP in predicting these latter two outcomes.

\section{Consent}

All persons gave their informed consent prior to their inclusion in the study.

\section{Conflicts of Interest}

The authors declare that they have no conflict of interest.

\section{Acknowledgments}

The authors acknowledge the technical assistance of Ms. Raija Isomäki and Ms. Anu Holopainen.

\section{References}

[1] R. T. Clarke, T. Jenyon, V. van Hamel Parsons, and A. J. King, "Neutropenic sepsis: management and complications," Clinical Medicine (London, England), vol. 13, no. 2, pp. 185-187, 2013.

[2] B. Suberviola, A. Marquez-Lopez, A. Castellanos-Ortega, C. Fernandez-Mazarrasa, M. Santibanez, and L. M. Martinez, "Microbiological diagnosis of sepsis: polymerase chain reaction system versus blood cultures," American Journal of Critical Care, vol. 25, no. 1, pp. 68-75, 2016.

[3] S. Hämäläinen, A. Juutilainen, I. Matinlauri et al., "Serum vascular endothelial growth factor in adult haematological patients with neutropenic fever: a comparison with Creactive protein," European Journal of Haematology, vol. 83, no. 3, pp. 251-257, 2009.

[4] Y. Sakr, C. Sponholz, F. Tuche, F. Brunkhorst, and K. Reinhart, "The role of procalcitonin in febrile neutropenic patients: review of the literature," Infection, vol. 36, no. 5, pp. 396407, 2008.

[5] M. Westwood, B. Ramaekers, P. Whiting et al., "Procalcitonin testing to guide antibiotic therapy for the treatment of sepsis in intensive care settings and for suspected bacterial infection in emergency department settings: a systematic review and cost-effectiveness analysis," Health Technology Assessment, vol. 19, no. 96, 2015.

[6] J. Zhang, Z. D. Hu, J. Song, and J. Shao, "Diagnostic value of presepsin for sepsis: a systematic review and meta-analysis," Medicine (United States), vol. 94, no. 47, article e2158, 2015.

[7] M. Mussap, A. Noto, M. Fravega, and V. Fanos, "Soluble CD14 subtype presepsin (sCD14-ST) and lipopolysaccharide binding protein (LBP) in neonatal sepsis: new clinical and analytical perspectives for two old biomarkers," Journal of 
Maternal-Fetal and Neonatal Medicine, vol. 24, Supplement 2, pp. 12-14, 2011.

[8] C. Chenevier-Gobeaux, D. Borderie, N. Weiss, T. MalletCoste, and Y. E. Claessens, "Presepsin (sCD14-ST), an innate immune response marker in sepsis," Clinica Chimica Acta, vol. 450, pp. 97-103, 2015.

[9] L. Pugni, C. Pietrasanta, S. Milani et al., "Presepsin (soluble CD14 subtype): reference ranges of a new sepsis marker in term and preterm neonates," PLoS One, vol. 10, no. 12, article e0146020, 2015.

[10] G. L. Su, K. Dorko, S. C. Strom, A. K. Nüssler, and S. C. Wang, "CD14 expression and production by human hepatocytes," Journal of Hepatology, vol. 31, no. 3, pp. 435-442, 1999.

[11] R. Landmann, W. Zimmerli, S. Sansano et al., "Increased circulating soluble CD14 is associated with high mortality in gram-negative septic shock," The Journal of Infectious Diseases, vol. 171, no. 3, pp. 639-644, 1995.

[12] H. Burgmann, S. Winkler, G. J. Locker et al., "Increased serum concentration of soluble CD14 is a prognostic marker in grampositive sepsis," Clinical Immunology and Immunopathology, vol. 80, no. 3, pp. 307-310, 1996.

[13] R. Landmann, A. M. Reber, S. Sansano, and W. Zimmerli, "Function of soluble CD14 in serum from patients with septic shock," The Journal of Infectious Diseases, vol. 173, no. 3, pp. 661-668, 1996.

[14] N. Hiki, D. Berger, C. Prigl et al., "Endotoxin binding and elimination by monocytes: secretion of soluble CD14 represents an inducible mechanism counteracting reduced expression of membrane CD14 in patients with sepsis and in a patient with paroxysmal nocturnal hemoglobinuria," Infection and Immunity, vol. 66, no. 3, pp. 1135-1141, 1998.

[15] R. Berner, B. Fürll, F. Stelter, J. Dröse, H. P. Müller, and C. Schütt, "Elevated levels of lipopolysaccharide-binding protein and soluble CD14 in plasma in neonatal early-onset sepsis," Clinical and Diagnostic Laboratory Immunology, vol. 9, no. 2, pp. 440-445, 2002.

[16] R. L. Kitchens and P. A. Thompson, "Impact of sepsis-induced changes in plasma on LPS interactions with monocytes and plasma lipoproteins: roles of soluble CD14, LBP, and acute phase lipoproteins," Journal of Endotoxin Research, vol. 9, no. 2, pp. 113-118, 2003.

[17] H. Aalto, A. Takala, H. Kautiainen, S. Siitonen, and H. Repo, "Monocyte CD14 and soluble CD14 in predicting mortality of patients with severe community acquired infection," Scandinavian Journal of Infectious Diseases, vol. 39, no. 6-7, pp. 596-603, 2007.

[18] B. B. de Aguiar, I. Girardi, D. D. Paskulin et al., "CD14 expression in the first $24 \mathrm{~h}$ of sepsis: effect of $-260 \mathrm{C}>\mathrm{T}$ CD14 SNP," Immunological Investigations, vol. 37, no. 8, pp. 752-769, 2008.

[19] J. J. Rios-Toro, M. Marquez-Coello, J. M. Alvarez et al., "Soluble membrane receptors, interleukin 6, procalcitonin and $\mathrm{C}$ reactive protein as prognostic markers in patients with severe sepsis and septic shock," PLoS One, vol. 12, no. 4, article e0175254, 2017.

[20] J. Wu, L. Hu, G. Zhang, F. Wu, and T. He, "Accuracy of presepsin in sepsis diagnosis: a systematic review and metaanalysis," PLoS One, vol. 10, no. 7, article e0133057, 2015.

[21] Z. Zheng, L. Jiang, L. Ye, Y. Gao, L. Tang, and M. Zhang, "The accuracy of presepsin for the diagnosis of sepsis from SIRS: a systematic review and meta-analysis," Annals of Intensive Care, vol. 5, no. 1, p. 48, 2015.
[22] V. Urbonas, A. Eidukaite, and I. Tamuliene, "The predictive value of soluble biomarkers (CD14 subtype, interleukin-2 receptor, human leucocyte antigen-G) and procalcitonin in the detection of bacteremia and sepsis in pediatric oncology patients with chemotherapy-induced febrile neutropenia," Cytokine, vol. 62, no. 1, pp. 34-37, 2013.

[23] E. Olad, I. Sedighi, A. Mehrvar et al., "Presepsin (sCD14) as a marker of serious bacterial infections in chemotherapy induced severe neutropenia," Iranian Journal of Pediatrics, vol. 24, no. 6, pp. 715-722, 2014.

[24] H. Koh, M. Aimoto, T. Katayama et al., "Diagnostic value of levels of presepsin (soluble CD14-subtype) in febrile neutropenia in patients with hematological disorders," Journal of Infection and Chemotherapy, vol. 22, no. 7, pp. 466-471, 2016.

[25] W. T. Hughes, D. Armstrong, G. P. Bodey et al., "2002 guidelines for the use of antimicrobial agents in neutropenic patients with cancer," Clinical Infectious Diseases, vol. 34, no. 6, pp. 730-751, 2002.

[26] R. C. Bone, R. A. Balk, F. B. Cerra et al., "Definitions for sepsis and organ failure and guidelines for the use of innovative therapies in sepsis," Chest, vol. 101, no. 6, pp. 1644-1655, 1992.

[27] M. M. Levy, M. P. Fink, J. C. Marshall et al., "2001 SCCM/ ESICM/ACCP/ATS/SIS international sepsis definitions conference," Intensive Care Medicine, vol. 29, no. 4, pp. 530-538, 2003.

[28] R. P. Dellinger, M. M. Levy, A. Rhodes et al., "Surviving sepsis campaign: international guidelines for management of severe sepsis and septic shock, 2012," Intensive Care Medicine, vol. 39, no. 2, pp. 165-228, 2013.

[29] M. Singer, C. S. Deutschman, C. W. Seymour et al., "The third international consensus definitions for sepsis and septic shock (sepsis-3)," Journal of the American Medical Association, vol. 315 , no. 8, pp. 801-810, 2016.

[30] C. L. Sprung, R. M. Schein, and R. A. Balk, “The new sepsis consensus definitions: the good, the bad and the ugly," Intensive Care Medicine, vol. 42, no. 12, pp. 2024-2026, 2016.

[31] S. Hämäläinen, A. Juutilainen, T. Kuittinen et al., "Serum amino-terminal pro-brain natriuretic peptide in hematological patients with neutropenic fever: a prospective comparison with C-reactive protein," Leukemia \& Lymphoma, vol. 51, no. 6, pp. 1040-1046, 2010.

[32] A. Juutilainen, S. Hämäläinen, K. Pulkki et al., "Biomarkers for bacteremia and severe sepsis in hematological patients with neutropenic fever: multivariate logistic regression analysis and factor analysis," Leukemia \& Lymphoma, vol. 52, no. 12, pp. 2349-2355, 2011.

[33] A. Juutilainen, M. Vänskä, K. Pulkki et al., "Pentraxin 3 predicts complicated course of febrile neutropenia in haematological patients, but the decision level depends on the underlying malignancy," European Journal of Haematology, vol. 87, no. 5, pp. 441-447, 2011.

[34] M. Vänskä, I. Koivula, S. Hämäläinen et al., "High pentraxin 3 level predicts septic shock and bacteremia at the onset of febrile neutropenia after intensive chemotherapy of hematologic patients," Haematologica, vol. 96, no. 9, pp. 1385-1389, 2011.

[35] A. K. Purhonen, M. Vänskä, S. Hämäläinen et al., "Plasma copeptin in the assessment of febrile neutropenia," Peptides, vol. 36, no. 1, pp. 129-132, 2012. 
[36] M. Vänskä, I. Koivula, E. Jantunen et al., "IL-10 combined with procalcitonin improves early prediction of complications of febrile neutropenia in hematological patients," Cytokine, vol. 60, no. 3, pp. 787-792, 2012.

[37] M. Vänskä, A. K. Purhonen, I. Koivula et al., "Soluble form of urokinase-type plasminogen activator receptor as a diagnostic and prognostic marker in hematological patients with neutropenic fever," Leukemia \& Lymphoma, vol. 55, no. 3, pp. 718-721, 2014.

[38] A. K. Purhonen, A. Juutilainen, M. Vänskä et al., "Human plasma cell-free DNA as a predictor of infectious complications of neutropenic fever in hematological patients," Infectious Diseases, vol. 47, no. 4, pp. 255-259, 2015.

[39] S. Bas, B. R. Gauthier, U. Spenato, S. Stingelin, and C. Gabay, "CD14 is an acute-phase protein," Journal of Immunology, vol. 172, no. 7, pp. 4470-4479, 2004.

[40] C. Chenevier-Gobeaux, E. Trabattoni, M. Roelens, D. Borderie, and Y. E. Claessens, "Presepsin (sCD14-ST) in emergency department: the need for adapted threshold values?," Clinica Chimica Acta, vol. 427, pp. 34-36, 2014.

[41] S. Masson, P. Caironi, C. Fanizza et al., "Circulating presepsin (soluble CD14 subtype) as a marker of host response in patients with severe sepsis or septic shock: data from the multicenter, randomized ALBIOS trial," Intensive Care Medicine, vol. 41, no. 1, pp. 12-20, 2015.

[42] T. Nagata, Y. Yasuda, M. Ando et al., "Clinical impact of kidney function on presepsin levels," PLoS One, vol. 10, no. 6, article e0129159, 2015.

[43] R. Poesen, A. Ramezani, K. Claes et al., "Associations of soluble CD14 and endotoxin with mortality, cardiovascular disease, and progression of kidney disease among patients with CKD," Clinical Journal of the American Society of Nephrology, vol. 10, no. 9, pp. 1525-1533, 2015.

[44] M. Behnes, T. Bertsch, D. Lepiorz et al., "Diagnostic and prognostic utility of soluble CD 14 subtype (presepsin) for severe sepsis and septic shock during the first week of intensive care treatment," Critical Care, vol. 18, no. 5, p. 507, 2014. 


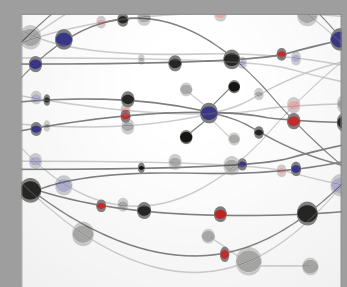

The Scientific World Journal
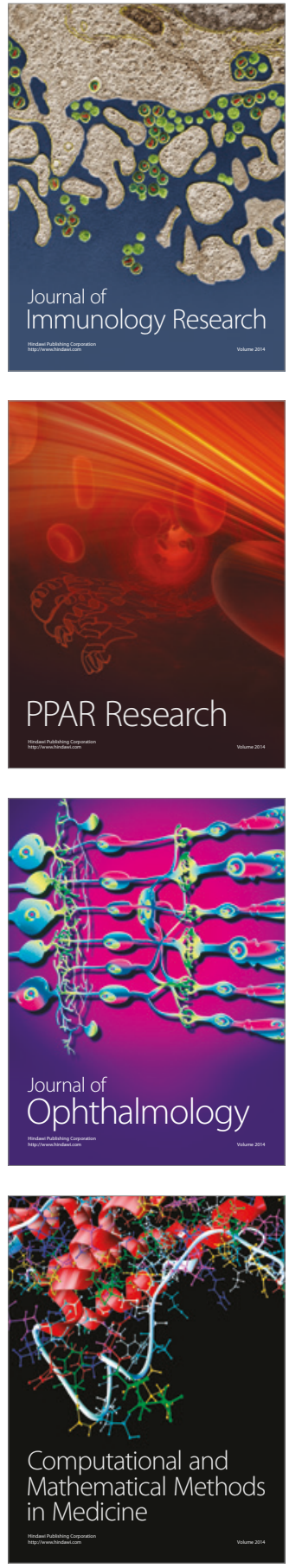

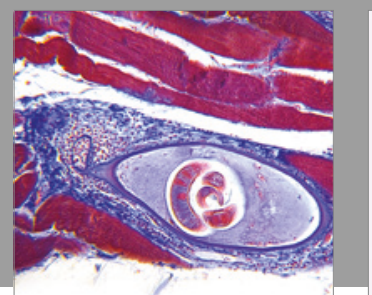

Gastroenterology Research and Practice
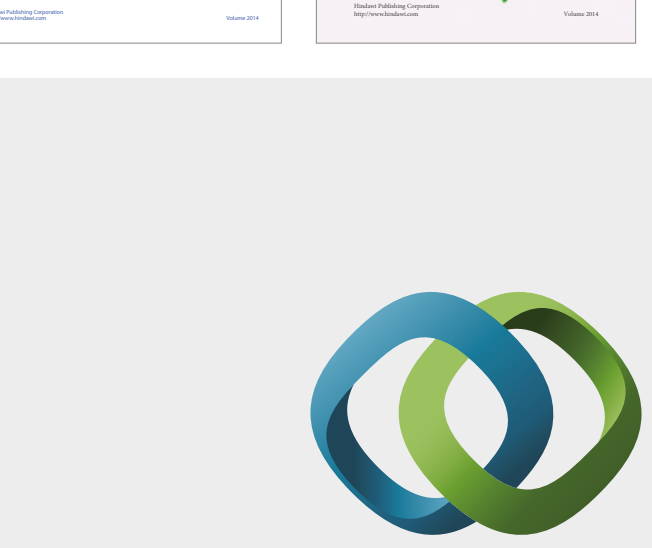

\section{Hindawi}

Submit your manuscripts at

https://www.hindawi.com
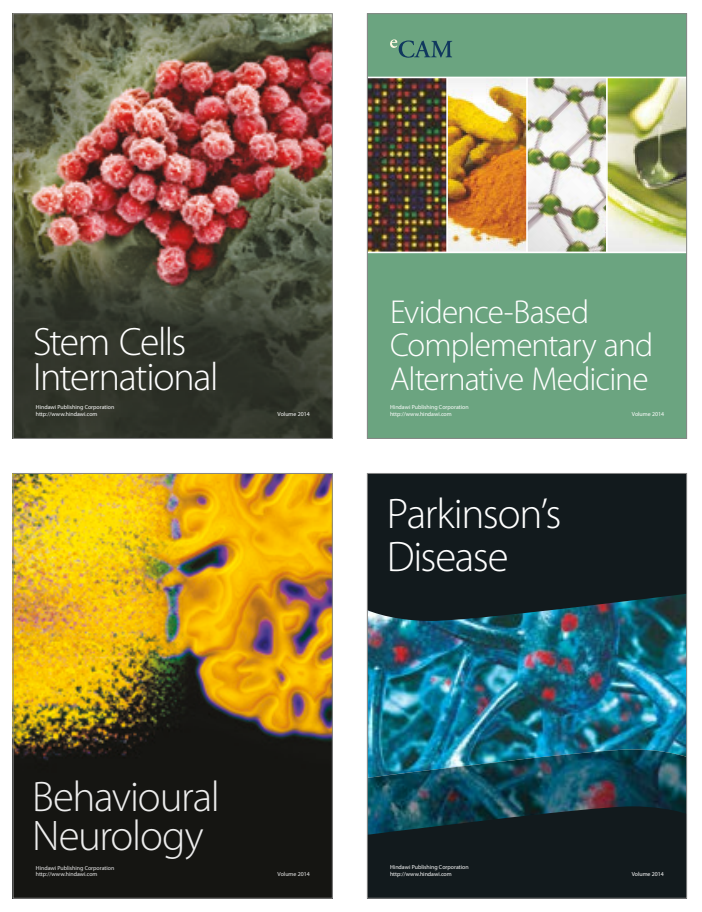
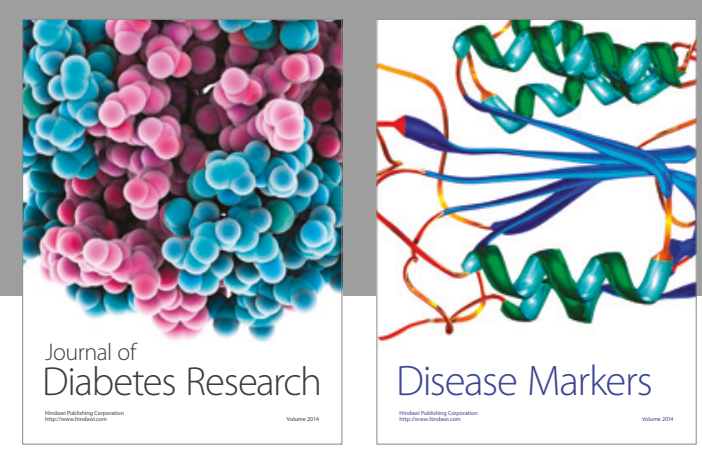

Disease Markers
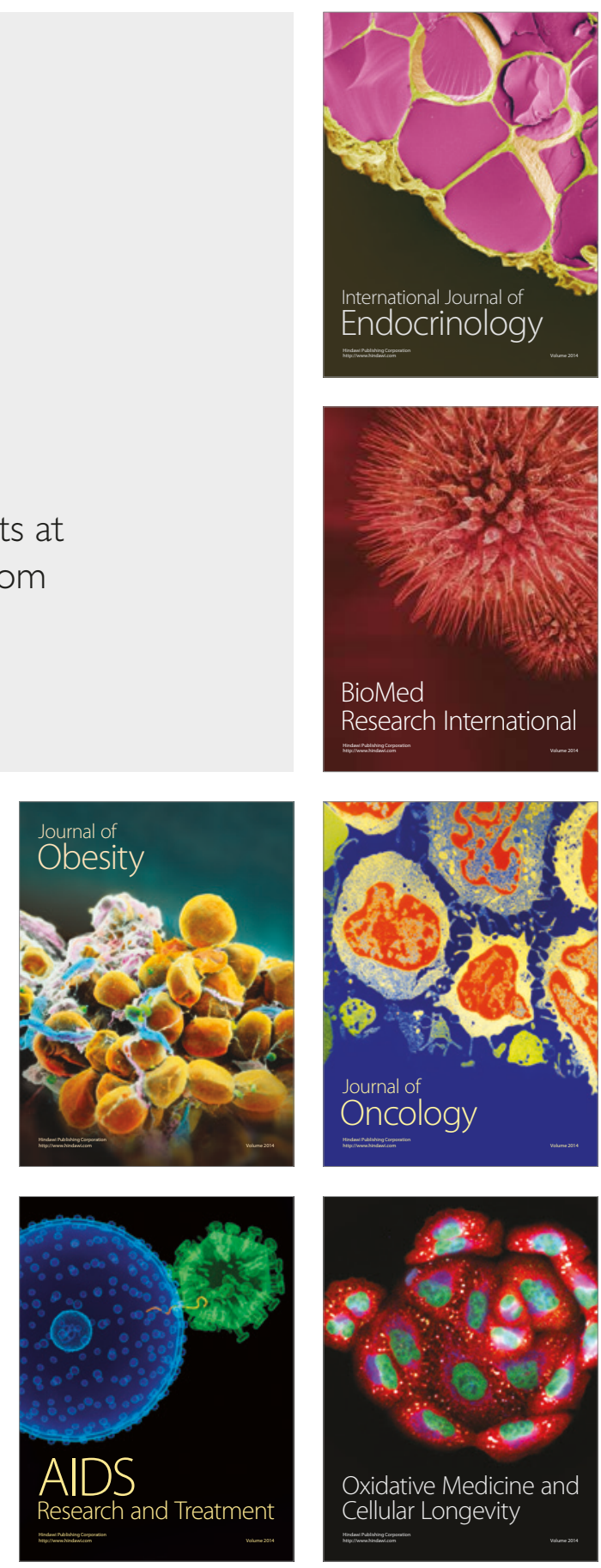